\title{
Design and Characterisation of Terabit/s Capable Compact Localisation and Beam-steering Terminals for Fiber-Wireless-Fiber Links
}

\author{
Ravinder Singh, Feng Feng, Yang Hong, Grahame Faulkner, Rushikesh Deshmukh, Guillaume Vercasson, \\ Olivier Bouchet, Periklis Petropoulos and Dominic O'Brien, Member, IEEE
}

\begin{abstract}
This paper presents the design, implementation and characterisation of compact fiber-wireless-fiber (FWF) localisation and beam-steering terminals, which provide a bi-directional free-space connection between fiber optic transceivers. The use of simple mirror based beam-steering, with high localisation accuracy in both steering axes enables ultra-high data rate transmission and practical indoor coverage. The auto-aligned terminals have a tracking accuracy of $00.02^{\circ}$, a full angle fieldof-view (FoV) of $50^{\circ}$, a tracking latency of $200 \mathrm{~ms}$ and work at wavelengths between $1300-1700 \mathrm{~nm}$. In an offline data processing experimental setup, $1 \mathrm{Tbit} / \mathrm{s}$ free-space transmission capability is demonstrated over a $2.54 \mathrm{~m}^{2}$ coverage area using ten wavelength division multiplexed PAM-4 signals. Further, full-duplex, realtime, data transmission using off-the-shelf small form-factor pluggable (SFP) optical transceivers is also demonstrated for Virtual Reality (VR) application. Here, using standard 10G SFP+ transceivers without the use of any pre/post optical amplification, the auto-aligned FWF terminals achieve $4.9 \mathrm{~m}^{2}$ localisation and $3.14 \mathrm{~m}^{2}$ bidirectional communications coverage over a range of $\sim 4 \mathrm{~m}$.
\end{abstract}

Index Terms-Optical Wireless Communication, Localisation and Tracking, Fiber-Wireless-Fiber, Beam-steering, Pencil Beams, Passive Optical Networks, Fiber Optic Transceivers.

\section{INTRODUCTION}

$\mathbf{F}$ IBER transmission systems have multi Tbit/s capability, making them ideal for data transportation in backhaul and access networks [1]. Here, passive optical networks (PON) have seen rapid deployment enabling availability of higher capacities to the end users [2]. Demand drives the development of radio and optical wireless access technologies, that are capable of delivering multi-Gbit/s to the end users over ranges of a few meters [3]-[6], for high bandwidth augmented and virtual reality applications.

Research on FWF terminals to create ultra-high data rate wireless links between ends of two fibers has gained increasing interest [7]-[9], allowing for end to end all optical networks.

R. Singh, R. Deshmukh, G. Faulkner and D. C. O'Brien are with the Department of Engineering Science, University of Oxford, UK, OX1 3PJ email: (ravinder.singh@eng.ox.ac.uk).

F. Feng is with the Key Laboratory of Opto-electronics Information Technology of Ministry of Education and Key Laboratory of Integrated Opto-electronic Technologies and Devices in Tianjin, School of Precision Instruments and Opto-electronics Engineering, Tianjin University, Tianjin 300072, China.

Y. Hong and P. Petropoulos are with the Optoelectronics Research Centre, University of Southampton, Southampton, SO17 1BJ, UK.

G. Vercasson is with B-COM Technology Research Institute, Rennes, France.

O. Bouchet is with Orange Labs, Rennes, France.
Broadly, the FWF terminals comprise a light collimator, beamsteering unit to enable user movement and a localisation \& tracking system to guide the beam-steering unit. Ultra-high data rate wireless communications, both Radio Frequency (RF) and optical, use narrow communication beams that must be steered to connect terminals, and the links formed are line of sight only. In the case of THz RF systems, optical fibres [10] are used to make data connections to terminals, which contain both baseband and RF processing stages. FWF links allow, over short-range, a direct connection between such optical fibres, removing much of the complexity of the RF alternative. FWF links are bidirectional by their nature, and transparent to modulation scheme and wavelength in the case of system presented in this work. Near term applications include VR and other latency critical applications where simple modulation and sufficient capacity to send uncompressed signals are advantageous. In the longer term multiple wireless links that combine capacity using line of sight approaches and reliability using sub $6 \mathrm{GHz}$ RF will be used to meet the exponentially growing demand for wireless connectivity.

Beyond Tbit/s data transmission has been demonstrated for FWF OWC systems [11]-[13], however, these demonstrations focused on fixed point-to-point links with no movement of the user terminals. In order to enable user movement within a certain coverage area, multi-Gbit/s OWC systems commonly rely on the use of fast steering mirrors (FSMs) [14]-[19], spatial light modulators (SLMs) [20] and passive diffraction gratings [8][9] for beam-steering. Compared to diffraction gratings, the FSMs have the capability to provide a wavelength independent wide indoor coverage, making them compatible with and transparent to passive WDM network. SLMs can provide point-to-multipoint connection at the base-station [21], but suffer added loss due to required optics for enhancing the beam-steering capability. Here, the focus is on the use of FSMs, although a combined FSM-SLM architecture has been demonstrated [21].

There have been various reports on the tracked OWC terminals which use FSMs. Practical implementation has proved demanding due to factors such as the need for an additional channel to transfer location information between terminals [14], time consuming 2D scanning for power maximisation [16], the need for large number of transceivers due to limited FoV [17], power inefficiency due to large beam footprint [19] and limited data-rate and transparency to PONs due to the use of free-space receivers [15]. Further, camera based localisation 
and tracking has been proposed in the literature [15][20][22]. This work is distinct as it uses two cost-effective ${ }^{1}$ cameras, one for coarse and one for fine tracking functions, and this allows low-cost tracking cameras with low pixel-counts to be used, with higher accuracy. Additionally, tracking is implemented at both access points and user terminals using this approach, which is important for minimising the alignment losses in a bi-directional and transparent FWF system. In addition to this measured tracking latency results are presented, an important factor for high mobility systems.

Aiming to mitigate these practical implementation challenges, we have designed FWF terminals, which use offthe-shelf dual-axis FSMs and cost effective small form-factor cameras for coarse and fine tracking, allowing compact integration of the beam-steering and localisation functionalities in a $15 \times 14 \times 13 \mathrm{~cm}$ housing. Recently, we have tested these FWF terminals using orthogonal frequency division multiplexed transmission [23]. This paper presents the design, implementation and characterisation of these FWF terminals, which provide compatibility with broad optical spectrum for high data rates, transparency to PON protocols, large indoor coverage, low tracking latency and high localisation accuracy to improve power efficiency. The system FoV, tracking accuracy, latency, link-loss, and localisation \& communications coverages are studied experimentally and an aberration correction mechanism is implemented. Additionally we report use in virtual reality experiment, and for Tbit/s transmission use 4-PAM scheme, thus reducing the complexity compared with the previous OFDM demonstration [23]. The results show that the terminals can provide beyond $1 \mathrm{Tbit} / \mathrm{s}$ capacities, and a localisation coverage of up to $4.9 \mathrm{~m}^{2}$. To the best of the authors' knowledge, this is the first report of fully automated FWF OWC terminals with such capabilities.

This paper is organised as follows: Section II describes the design and control of the compact FWF terminals for auto alignment. The FoV, link-loss, aberrations and tracking latency are characterised in section III. Section IV presents the experimental results for ultra-high data transmission rate capability and section $\mathrm{V}$ presents real-time, full-duplex data transmission results obtained through use of SFP transceivers. Section VI presents the concluding remarks.

\section{Description of Design AND CONTROL}

\section{A. Design}

Fig. 1 shows the system schematic and Table I lists the components of the FWF terminals. The communications signal, represented by yellow lines, is collimated out/into fibers with an optical collimator and steered using dual-axis steering mirrors. The red and blue lines represent the infrared localisation beacons provided by IR tags. The optical information signal and localisation beacon light are separated through the use of dichroic filters (DF1 \& DF2). The localisation sub-system uses two board cameras, one for coarse tracking (CAM1 \& CAM3) and second for fine tracking (CAM2 \& CAM4). Further, use of optical band-pass filters (BF1 \& BF2) minimises the possible

${ }^{1} \mathrm{~A}$ factor of more than ten times cheaper than the one used in [20]

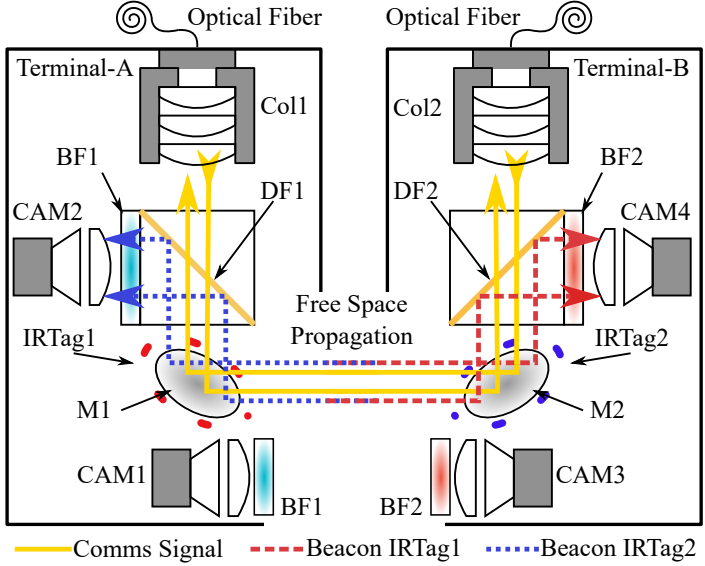

Fig. 1. Schematic of the proposed FWF terminals.

interference to the localisation system from unwanted visible and infrared illumination sources.

The localisation sub-system uses two cameras to provide both wide FoV and higher accuracy. The wide FoV camera allows terminals to be located. The higher accuracy narrow FoV camera allows higher precision steering such that misalignment power loss is small. The gold-coated mirrors (M1 \& M2) provide dual-axis beam steering with up to $\pm 50^{\circ}$ optical deflection per axis and a steering resolution less than $5 \mu \mathrm{rad}$. The mirrors have a large angle step $\left(20^{\circ}\right.$ step) response time of $\sim 7.5 \mathrm{~ms}$ and a small angle step $\left(0.1^{\circ} \mathrm{step}\right)$ response time of $\sim 1.4 \mathrm{~ms}$. These are sufficient for nomadic operations. It must be noted that the use of mirror based beam steering allows point-to-point links only. This means that access point can serve only one user at any one particular time. However, multi-user access can be supported by means of TDMA. Given the high data-rates that can be achieved (see Section IV), the TDMA approach with a short time-slot will be quite realistic and efficient to support multiple users through these terminals. Such a use case would also rely on RF based systems to provide reliable low-data rate connectivity (such as $60 \mathrm{GHz}$ $\mathrm{RF}$ wireless works together with WiFi).

TABLE I

FWF TERMINAL COMPONENT DESCRIPTION.

\begin{tabular}{|c|c|c|}
\hline Notation & Description & Part Number \\
\hline BF1 and BF2 & $\begin{array}{c}\text { Optical Bandpass } \\
\text { Filters }\end{array}$ & $\begin{array}{c}\text { Edmund Optics \#84-777 } \\
\text { and \#84-779 }\end{array}$ \\
\hline Col1 and Col2 & Fiber Collimators & $\begin{array}{c}\text { ThorLabs } \\
\text { TC06APC-1550 }\end{array}$ \\
\hline $\begin{array}{c}\text { CAM1 and } \\
\text { CAM3 }\end{array}$ & $\begin{array}{c}\text { Coarse Tracking } \\
\text { Board Cameras }\end{array}$ & PixyCam 1 ${ }^{\text {st }}$ Generation \\
\hline $\begin{array}{c}\text { CAM2 and } \\
\text { CAM4 }\end{array}$ & $\begin{array}{c}\text { Fine Tracking Board } \\
\text { Cameras }\end{array}$ & PixyCam 1 ${ }^{\text {st }}$ Generation \\
\hline DF1 and DF2 & Dichroic Filters & Edmund Optics \#69-907 \\
\hline $\begin{array}{c}\text { IRTag1 and } \\
\text { IRTag2 }\end{array}$ & $\begin{array}{c}\text { localisation LED } \\
\text { Rings }\end{array}$ & $\begin{array}{c}\text { Ushio SMT800 and } \\
\text { SMT890 }\end{array}$ \\
\hline M1 and M2 & $\begin{array}{c}\text { Dual-axis Steerable } \\
\text { Mirror }\end{array}$ & OptoTune MR-15-30 \\
\hline
\end{tabular}

The coarse tracking cameras CAM1 and CAM3 were equipped with $2.1 \mathrm{~mm}$ focal length imaging lens (Edmund Optics 55-569) which resulted in a FoV of $\pm 50^{\circ}$ horizontally 
and $\pm 30^{\circ}$ vertically for the $3.89 \times 2.43 \mu \mathrm{m}$ sensor fitted in the pixycam. This can be easily estimated using the following equation,

$$
\mathcal{F}(H, V)=2 * \tan ^{-1}\left(\frac{S(H, V) / 2}{F_{L}}\right),
$$

where $\mathcal{F}(H, V)$ is the horizontal and vertical field of view (FoV), calculated from the lens focal length $F_{L}$ and the width $S(H)$ and length $S(V)$ of the camera sensor, respectively. The pixycam sensor has $1280 \times 800$ pixels. However, the on-board post-processing results in $320 \times 200$ pixel frames at the $\mathrm{I}^{2} \mathrm{C}$ output. This gives approximately $0.3^{\circ}$ per pixel resolution for the coarse tracking cameras. In order to choose the imaging lens for the fine tracking CAM2 and CAM4, the required pointing accuracy was investigated to ensure low loss coupling with fiber collimators. This involved measuring additional power loss due to misalignment when the transmitter is pointed at a static receiver, and the receiver pointed at a static transmitter, for a link distance of $3.5 \mathrm{~m}$. The results are shown in Fig. 2. It can be seen that a $\pm 0.01^{\circ}$ misalignment in pointing results in an additional loss of approximately $3 \mathrm{~dB}$ relative to maximum transmission. Therefore, a $50 \mathrm{~mm}$ focal length imaging lens (Edmund Optics 59-781) was used for fine tracking cameras, which resulted in a FoV of $\pm 2^{\circ}$ horizontally and $\pm 1.1^{\circ}$ vertically, leading to approximately $0.0125^{\circ}$ per pixel resolution. The coarse and fine tracking camera imaging lenses have an entrance aperture of $17 \mathrm{~mm}$ and $27 \mathrm{~mm}$, respectively.

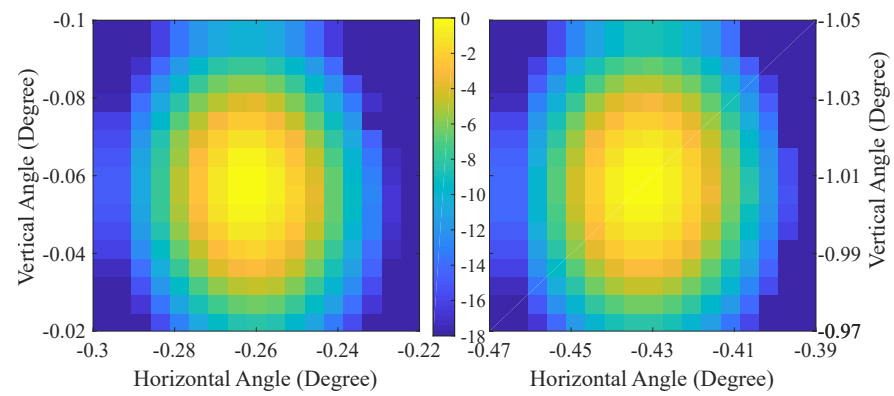

Fig. 2. Additional loss $(\mathrm{dB})$ due to pointing misalignment. Left figure shows terminal-1 pointing, terminal-2 static. Right figure shows terminal-2 pointing, terminal-1 static.

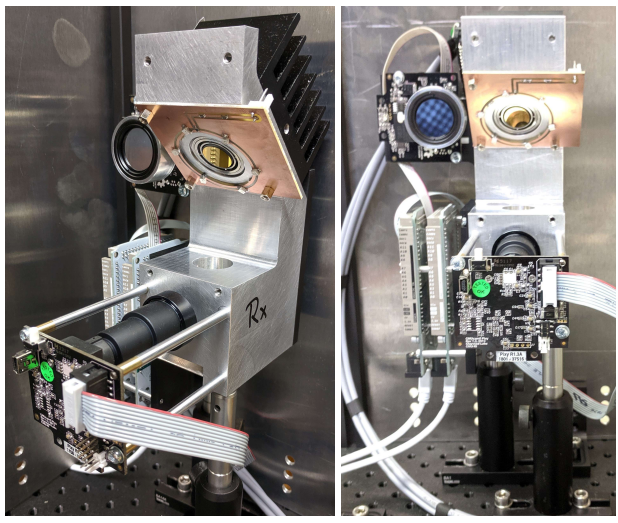

Fig. 3. Fully assembled FWF terminals.

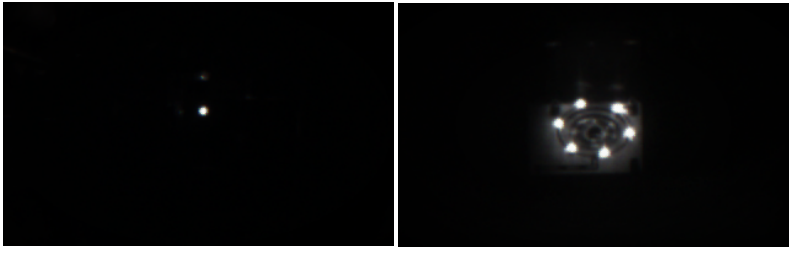

Fig. 4. The IR beacon as seen by coarse (left) and fine (right) tracking cameras at 3 meter distance.

Fig. 3 shows the assembled units. The communication and localisation components are assembled with a metallic housing which is approximately $15 \times 14 \times 13 \mathrm{~cm}$. The IR tag PCBs are mounted on the same surface where the $2 \mathrm{D}$ steerable mirrors are affixed. Each PCB contains six IR LEDs forming a ring of $35 \mathrm{~mm}$ diameter, each LED separated by $17.5 \mathrm{~mm}$. This enables the fine tracking camera to image a complete LED ring at a minimum link distance of $0.5 \mathrm{~m}$ and also resolve the $17.5 \mathrm{~mm}$ spatially separated LEDs at the opposite terminal for a link distance of up to 6 meters on bore sight. The IR LED ring is placed around the beam steering mirror so that the position of the centre of the mirror can be estimated. Fig. 4 shows images of the IR beacon seen by a coarse and fine tracking camera, respectively at a link distance of $3.5 \mathrm{~m}$. The IR beacon appears as a single source to the coarse tracking camera due to its wide FoV and the fine tracking camera is able to resolve the $17.5 \mathrm{~mm}$ spatially separated LEDs. The fine tracking cameras provide the position of each LED in a frame, from which an accurate position of the centre of the opposite IR ring or the opposite steering mirror is estimated. The measured optical power from the $810 \mathrm{~nm}$ and $890 \mathrm{~nm}$ LED rings, which use DC power supply, was approximately $0.43 \mathrm{~mW} / \mathrm{cm}^{2}$ and $0.71 \mathrm{~mW} / \mathrm{cm}^{2}$ at $100 \mathrm{~mm}$ distance. These power densities are within the Class-1 eyesafe limits estimated following the IEC60825-1 standard. The LEDs (Ushio SMT800 and SMT890) in the IR rings have a rise-time $\leq 200 \mathrm{~ns}$, which makes it possible to drive these sources using frequency or intensity modulators as in [22]. This could potentially allow identification of user terminals and enable multi-user tracking.

\section{B. Terminal Control}

The terminals can be controlled through a microcontroller or a PC. During the characterisation, terminals were controlled with a PC running MATLAB. The board cameras (Pixycams) stream object (IRTag) information in terms of it's image size and centre on the sensor in pixels to Arduino Due boards at 50 frames per second (fps) rate via $\mathrm{I}^{2} \mathrm{C}$ interface. This information is acquired by the controller $\mathrm{PC}$ connected to the Arduino boards via USB connection for further processing to obtain yaw and pitch angles for the beam-steering mirrors. Once the angles have been estimated, MATLAB commands are sent to the mirror driver boards (provided by OptoTune) via USB connection to tilt the mirrors for terminal alignment.

The terminals are aligned in a two-step localisation and steering procedure, which is illustrated by a flowchart shown in Fig. 5. In the first step, rough positioning information 
of the opposite terminal is obtained by the coarse tracking camera. The coarse tracking positional information is converted to horizontal and vertical coarse link angles $\alpha_{H}$ and $\alpha_{V}$, respectively, using (2), where $O_{C T}(H, V)$ represent the horizontal or vertical centre pixels of coarse tracking camera frames, $I_{C T}(H, V)$ is the horizontal or vertical pixel numbers representing the centre of the object's image in a frame acquired from the coarse tracking cameras, and $\delta_{C T}(H, V)$ is the measured horizontal or vertical resolution of the coarse tracking cameras in degrees per pixel. The horizontal and vertical steering angles of the mirror at this point are given as $0.5 \alpha_{H}$ and $0.5 \alpha_{V}$, respectively.

$$
\alpha(H, V)=\left\{O_{C T}(H, V)-I_{C T}(H, V)\right\} \delta_{C T}(H, V)
$$

The coarse alignment positions the tracked IR beacon into the FoV of the fine tracking cameras, which then estimates finer mirror steering angles. In this step, the coordinates of the six LEDs are obtained from the fine tracking camera at a $50 \mathrm{fps}$ rate. Then, through the use of elliptical fitting

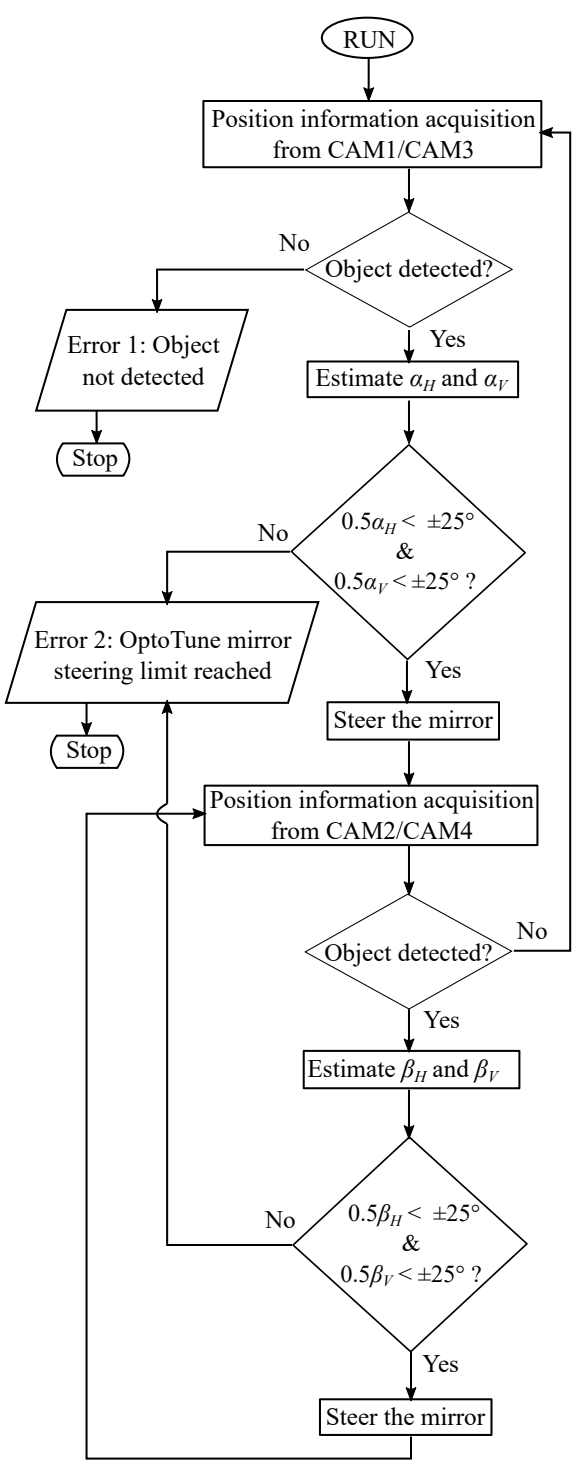

Fig. 5. Localisation and steering process for terminal alignment.
MATLAB function, the centre of the LED ring and hence the centre of the opposite mirror is estimated. The ring's centre coordinates are then converted to more precise link angles $\beta_{H}$ and $\beta_{V}$, respectively, using (3), where $\theta(H, V)$ represents the horizontal or vertical alignment calibration coefficient. Post fine tracking the horizontal and vertical steering angles of the mirror are given as $0.5 \beta_{H}$ and $0.5 \beta_{V}$, respectively. At this point the two terminals are aligned and up-link/down-link data transmission can take place.

$$
\beta(H, V)=\left\{O_{F T}(H, V)-I_{F T}(H, V)\right\} \delta_{F T}(H, V)+\theta(H, V)
$$

\section{CharACTERISATION OF LOCALISATION SYSTEM: FOV, LINK-LOSS, ABERRATIONS AND LATENCY}

The FoV and link-loss evaluation was carried out using the experimental setup shown in Fig. 6. The FWF terminals were placed on optical tables of same height. The freespace distance between the terminals was $3.5 \mathrm{~m}$. One of the terminal was affixed to the optical table and other terminal was rotated with the use of a rotation stage (driven by Newport ESP300). A 1550nm SFP+ transceiver module (Finisar FTLX1871M3BCL) was used as an optical source, which was driven by a bit error ratio tester (Spectronix Eye-BERT). A single mode fiber (SMF) connects the FWF terminals to SFP+ module and optical power meter (Newport 1919-R). A variable optical attenuator (VOA15-APC) was used between the BERT and transmitting terminal to control the output power.

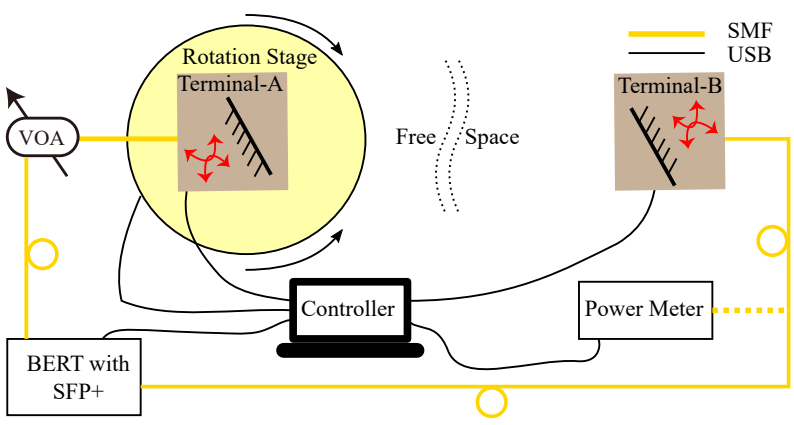

Fig. 6. Experimental setup for FoV and aberration evaluation.

The FoV and operation of localisation and steering system was inspected by separate horizontal and vertical rotation of one terminal, while the other terminal remained static at approximately $0^{\circ}$ steering angle. The transmit optical power was $0 \mathrm{dBm}$ by default. Auto-alignment was performed using the localisation system and the received power $(\mathrm{dBm})$ was recorded for every degree of terminal rotation both with and without the use of aberration correction. The results are shown in Fig. 7 for both the terminals, which show that the bore-sight loss is approximately $7 \mathrm{~dB}$, resulting from beam divergence, localisation limited beam steering accuracy and the collimation losses. In addition, aberrations in the localisation system lead to significant link loss as the link angle increases at any one terminal either in the horizontal or the vertical plane.

The localisation error due to aberrations at each terminal were measured in degrees for different link angles in both horizontal and vertical steering axes, which are shown in Fig. 8 


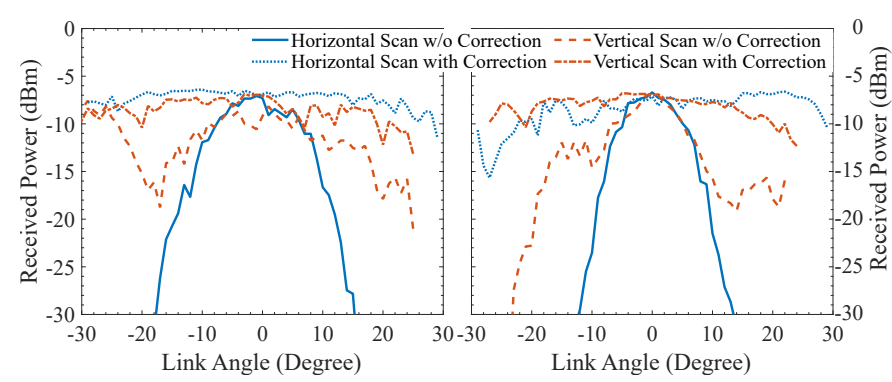

Fig. 7. Link-loss (dB) vs link-angle for the two FWF terminals. The solid curves represent received power without aberration corrections and the dashed curves with aberration correction.

in the form of steering angle correction for one of the terminals. The steering angle correction is measured by comparing the manual alignment angles with the automated alignment angles. These results show that the aberrations increase with increasing link angles, leading to positioning errors as high as $0.1^{\circ}$. The results also show that the steering in the horizontal and vertical planes is cross-coupled. These aberrations could potentially be generated by multiple factors, such as axial misalignment between mirror and the fine tracking camera, cross-coupling between the mirror rotation axis, etc. It has not been possible to link these aberrations to one particular source. In this work, $2^{\text {nd }}$ order polynomial equations of the form $\Delta \beta(H, V)=c_{1} \beta^{2}(H, V)+c_{2} \beta(H, V)+c_{3}$ were used to relate derived position from the localisation system and link establishing steering angles. The constants $c_{1}, c_{2}$ and $c_{3}$ were obtained by applying polynomial fit to the measured steering angle correction for every link angle. The results in Fig. 7 show that the aberration correction significantly improves the received power and reduces the link loss by more than $20 \mathrm{~dB}$ for a link angle of $\pm 20^{\circ}$, which has been used as an example.

Although coarse tracking provides a $\mathrm{FoV}$ of $\pm 50^{\circ}$ horizontally and $\pm 30^{\circ}$ vertically, the FoV of the assembled terminals was limited to $\pm 29^{\circ}$ horizontally and $\pm 25^{\circ}$ vertically. The FoV in the horizontal plane was limited by the ellipticity of the IR Tags making it difficult for the fine tracking camera to resolve the elements of the LED rings for link angles greater than $\pm 29^{\circ}$ horizontally. The FoV in the vertical plane was limited due to the position of the fine tracking camera, which blocked the communication signal for link angles greater than $+25^{\circ}$ vertically. The location of the fine tracking camera can be changed to fully access the available coverage in vertical plane. In the horizontal plane the coverage may be enhanced by further improvements in the design of the localisation system and software.

The tracking latency was studied by manually translating Terminal-B in the lateral direction. The terminals continuously and independently tracked each other. The received power and link angle was monitored during the translation time. The received power was measured and recorded with a power meter, whereas the link-angle was estimated and recorded from the localisation \& tracking system. The results are shown in Fig. 9, which show that for one lateral translation of Terminal$\mathrm{B}$, the power is down for approximately 1.3 seconds (see main figure), during which the Terminal-B, as it is moved,
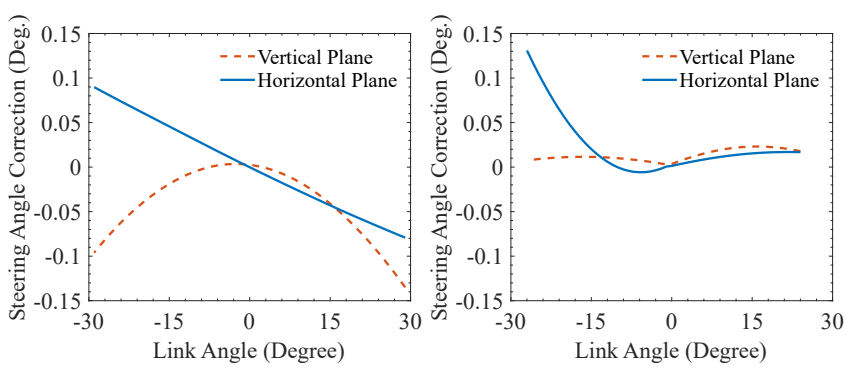

Fig. 8. Steering angle correction (Degree) for one of the terminals for steering in horizontal plane (left) and vertical plane (right).

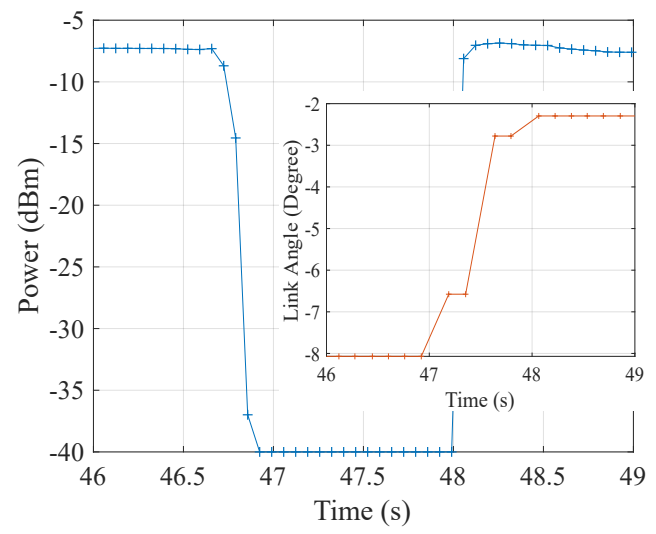

Fig. 9. The time versus received power plot for the manual translation of the Terminal-B in lateral direction with respect to Terminal-A. The inset shows time versus link angle for the Terminal-B, to study the terminal tracking latency.

tries to track Terminal-A six times (see inset), while the link angle changed from roughly -8 to $-2.3 \mathrm{deg}$. This gives an average tracking time of $216 \mathrm{~ms}$. Similar tracking behaviour and latency was observed on Terminal-A, as each terminal must track the other to maintain a link. The latency was further confirmed within the MATLAB by recording the time taken to run the coarse $(\sim 50 \mathrm{~ms})$ and fine tracking codes $(\sim 150 \mathrm{~ms})$. This latency is sufficiently low to support nomadic or quasistationary terminals. However, depending on the required level of mobility, this latency must be reduced. To do so faster sensor or a different approach to tracking will be required. This is a subject of further research. The measured characteristics of the FWF terminals are summarised in Table II.

TABLE II

FWF TERMINAL FEATURES AND SPECIFICATIONS.

\begin{tabular}{|c|c|}
\hline Operating FoV & $\pm 25^{\circ}$ \\
\hline Tracking Resolution (Measured) & $\begin{array}{l}0.021^{\circ} \text { horizontally and } 0.014^{\circ} \\
\text { vertically }\end{array}$ \\
\hline Communications Wavelngth & 1300-1730nm (Bidirectional) \\
\hline Fiber Input/Output & SMF 28 - APC connector \\
\hline Free Space Beam Diameter & $1.12 \mathrm{~mm}$ at the collimator output \\
\hline $\begin{array}{c}\text { Communication Beam } \\
\text { Divergence }\end{array}$ & $0.101^{\circ}$ full anlge \\
\hline Tracking Latency per Terminal & $216 \mathrm{~ms}$ \\
\hline Tracking Beacon Wavelength & $800 \mathrm{~nm}$ and $890 \mathrm{~nm}$ \\
\hline
\end{tabular}




\section{TBIT/S TRANSMISSION EXPERIMENT}

\section{A. Experiment layout}

Data transmission experiments in different positioning scenarios were conducted to experimentally verify the design of the presented FWF terminals, as illustrated in the layout shown in Fig. 10. The location of beam-steering terminal-A was fixed on an optical bench and terminal-B, sitting on another optical bench, was moved along the lateral direction to four equally spaced locations. The vertical distance between the terminals was 3.5 metres. For every location of terminal-B, both of the mirror-based beam steering units had to adjust their steering angles to establish the fibre-wireless-link. Due to space limitations, experimental tests for the lateral displacement up to $\sim 0.9$ metres from the origin of the localisation FoV were performed. This results in a $2.54 \mathrm{~m}^{2}$ full coverage, due to the symmetrical nature of the optical localisation system.

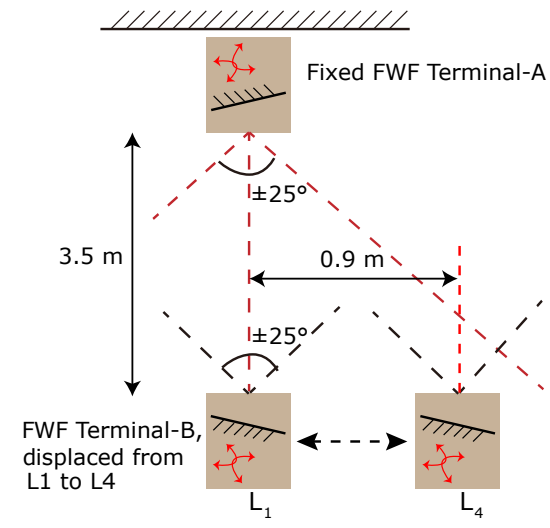

Fig. 10. Experimental layout for testing $1 \mathrm{Tbit} / \mathrm{s}$ transmission over the FWF terminals.

\section{B. Tbit/s tests and results}

Fig. 11 shows the data transmission components. Ten standard ITU 100-GHz-spaced wavelengths from $1546.12 \mathrm{~nm}$ to $1553.33 \mathrm{~nm}$ were used as optical carriers to achieve the aggregate data rate of 1 Terabit/s. A Mach-Zehnder modulator driven by an arbitrary waveform generator (AWG) that generated $100 \mathrm{~Gb} / \mathrm{s}$ Nyquist PAM-4 signals modulated each of the ten wavelengths. The odd and even WDM channels of the modulated optical signals were demultiplexed by a Finisar waveshaper and were de-correlated by using different fibre delays. Then they were combined and amplified by a booster erbium-doped-fiber amplifier (EDFA). In order to ensure eyesafe operation, the output optical power at the transmitting end was set to $7 \mathrm{dBm}$ (i.e. $3 \mathrm{~dB}$ below the eys-safety limit at $1550 \mathrm{~nm}$ as per the IEC60825-1 standard). At the receiving end, a pre-amplifying EDFA was employed and a tunable optical bandpass filter (OBPF) was used to individually select each of the WDM channels. The power of the received optical signal can be varied by a variable optical attenuator before it is detected by a photodiode. The converted electrical signals are then captured by an $80 \mathrm{GS} / \mathrm{s}$ real-time oscilloscope and processed offline. A decision feedback equalizer (DFE) was used to recover the PAM-4 signals and the bit error ratios (BERs) were estimated via error counting.

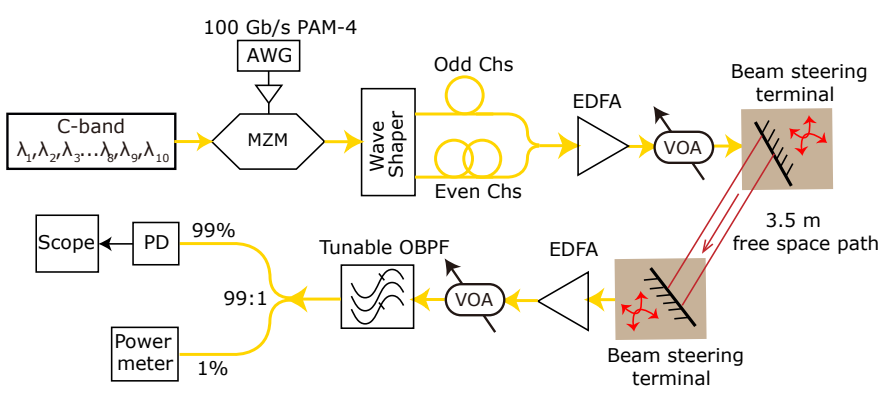

Fig. 11. Experimental setup for testing $1 \mathrm{Tbit} / \mathrm{s}$ transmission over the FWF terminals.

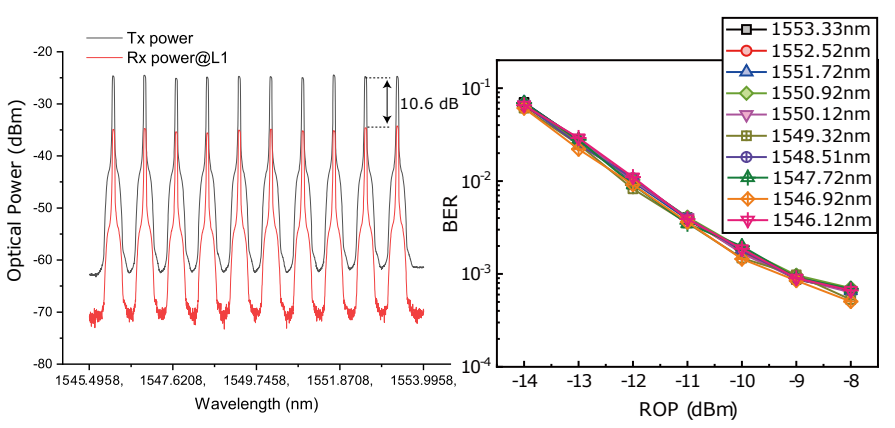

Fig. 12. The transmitted and received optical spectra of the 10 WDM channels (left), measured BER curves versus received optical power (ROP) for all WDM channels (right) for optimal alignment at location L1.

The 3-dB bandwidth of the MZM and PD were around 20 $\mathrm{GHz}$ and $30 \mathrm{GHz}$, respectively. The passband bandwidth of the OBPF was $0.8 \mathrm{~nm}$. Furthermore, the $7-\mathrm{dBm}$ launched power corresponds to the total power of all ten WDM channels. Initial data transmission tests at the aggregate data rate of 1 Terabit/s were performed when FWF terminal-B was at location L1. In this case, terminals were more or less on boresight with almost no lateral displacement, as illustrated in Fig. 10. The alignment of the indoor OWC link was manually optimized through 2D scans of both mirrors one after another and back and forth with a fine resolution of $0.01^{\circ}$ to identify the minimum link loss. Fig. 12(left) shows the measured transmitted and received optical spectra of the 1 Terabit/s WDM signal. An optical link loss of $\sim 10.6 \mathrm{~dB}$ was measured. Fig. 12(right) shows the measured BER curves versus received optical power for all WDM channels over the OWC link. A BER below the $7 \%$ forward-error-correction (FEC) threshold $\left(3.8 \times 10^{-3}\right)$ for all WDM channels was achieved. It can be seen that all the WDM channels of the OWC link have almost identical BER performance at all the received optical power values. This is due to the wavelength transparent nature of the mirror-based beam steering terminals.

To further investigate the indoor OWC system under tracking and localization, mirror terminal $\mathrm{B}$ was moved between four discrete locations, as illustrated in Fig. 10. At each location, OWC links were first established by the localisation system and were subsequently manually optimized. For each case of the tracked and optimised alignment, optical link loss was measured and the BERs for each of the ten PAM-4 WDM transmissions were evaluated. Fig. 13(left) shows the BER measurements at $-10 \mathrm{dBm}$ received optical power for all WDM 


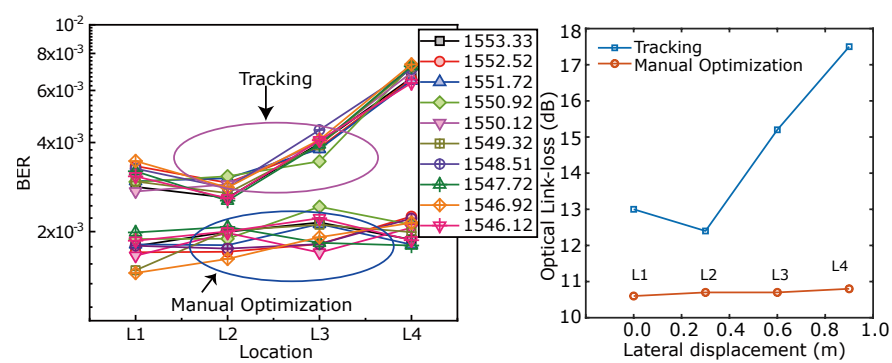

Fig. 13. At the four discrete locations, for both OWC links established by the tracking system and manually optimized OWC links, (left) BER curves of all WDM channels at $-10 \mathrm{dBm}$ received optical power, and (right) corresponding optical link loss.

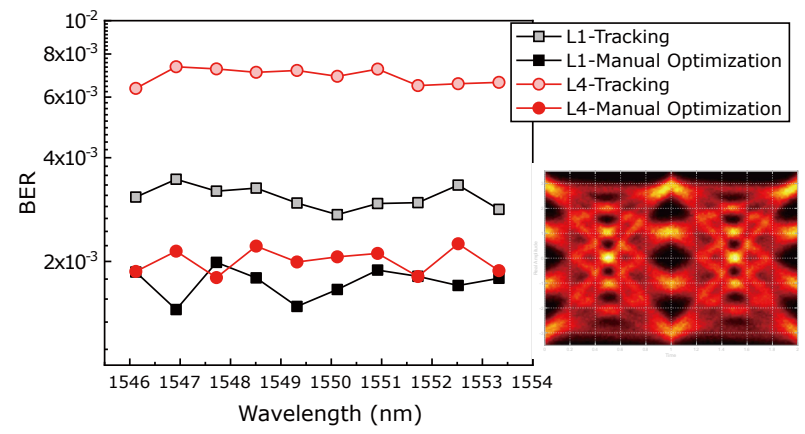

Fig. 14. BER curves (left) of all the WDM channels at location L1 and location L4 for both tracking established OWC links and manuallly optimized OWC links at $-10 \mathrm{dBm}$ received optical power. A typical $100 \mathrm{~Gb} / \mathrm{s}$ PAM-4 eye diagram (Right) at $-9 \mathrm{dBm}$ ROP for the manually optimized OWC link at location 1 .

channels on both localisation established links and further manually optimized links. Fig. 13(right) shows the measured optical losses for both the tracked links and optimized links at each location. It is observed that the optimal alignment with manual steering provides better BER than the auto-alignment, especially as the lateral distance increases. This is due to the $0.01^{\circ}$ difference between the steering accuracy of the localisation and optimal alignment scenarios. However, it must be noted that the manual alignment can take several hours in comparison to tracked alignment. Further optimisation of the tracking system could potentially overcome this additional power loss. More discussion on this is presented in Section V.

Fig. 14 compares the BER at $-10 \mathrm{dBm}$ received optical power versus wavelengths of all WDM channels for both the localisation established links and manually optimized links respectively at location L1 and location L4. It indicates that the data transmission performance of different WDM channels is similar at each position and wavelength. A typical PAM-4 eye diagram at $100 \mathrm{~Gb} / \mathrm{s}$ is also shown in Fig. 14.

\section{REAL-TIME, Full-DupleX TRANSMisSiON}

A 10G SFP+ transceiver module (FTLX1871M3BCL) which provides $0 \mathrm{dBm}$ output power at the transmitter was used for real-time data transmission experiments. The receiver sensitivity was determined using a free-space $10.3 \mathrm{Gbit} / \mathrm{s}$ data transmission link between terminals $3.5 \mathrm{~m}$ apart. The transmit power was reduced through the use of a VOA (VOA15-APC), leading to reduction in the received power and degradation of
BER. A plot of BER vs received power is shown in Fig. 15. It can be seen that the receiver has a sensitivity of $-27 \mathrm{dBm}$ for a BER of approximately $10^{-9}$, giving a link budget of 27 $\mathrm{dB}$ with a $0 \mathrm{dBm}$ transmission power.

The terminals were placed on separate trolleys at roughly $1 \mathrm{~m}$ height and the distance between the terminals was increased and data transmission was established through tracking for different link distances. The link distance vs received power results are shown in Fig. 16. It can be seen that the localisation system can provide bore-sight coverage of up to $8 \mathrm{~m}$ with a link margin of at least $1 \mathrm{~dB}$, maintaining $10.3 \mathrm{Gbit} / \mathrm{s}$ transmission. The link-loss increases with the link distance partly due to beam divergence and the limited accuracy of localisation system. Given that optical power can be increased to $10 \mathrm{dBm}$ at the transmitter (i.e. to the eye-safety limit), beyond $8 \mathrm{~m}$ transmission can be established by means of optical amplification, which can also be used at the receiver side to further increase the link-budget. Fig. 16 also shows the received power vs link distance curve for optimally aligned terminals. The results show that the optimal alignment can reduce the link losses by up to $10 \mathrm{~dB}$ for a link distance of $8 \mathrm{~m}$ and can further increase link distance significantly. However, as previously mentioned the optimal alignment with manual $2 \mathrm{D}$ scanning of the steering mirrors is impractical due to time inefficiency. Operation of a $1310 \mathrm{~nm}$ transmission was also investigated using another SFP+ transceiver, where an extra $2 \mathrm{~dB}$ loss was observed for this shorter wavelength optical signal.

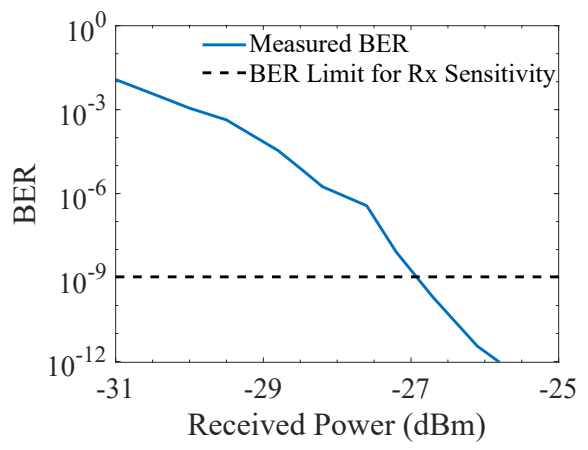

Fig. 15. BER performance of FTLX1871M3BCL SFP+ module for a 10.3 Gbit/s free-space transmission.

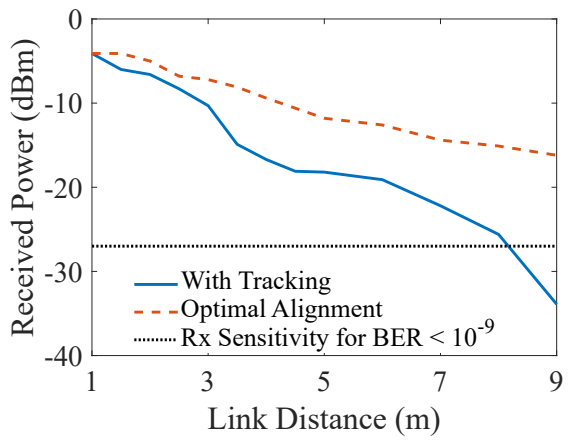

Fig. 16. Bore sight communication range of FWF terminals with SFP+ based $10.3 \mathrm{Gbit} / \mathrm{s}$ free-space transmission. 

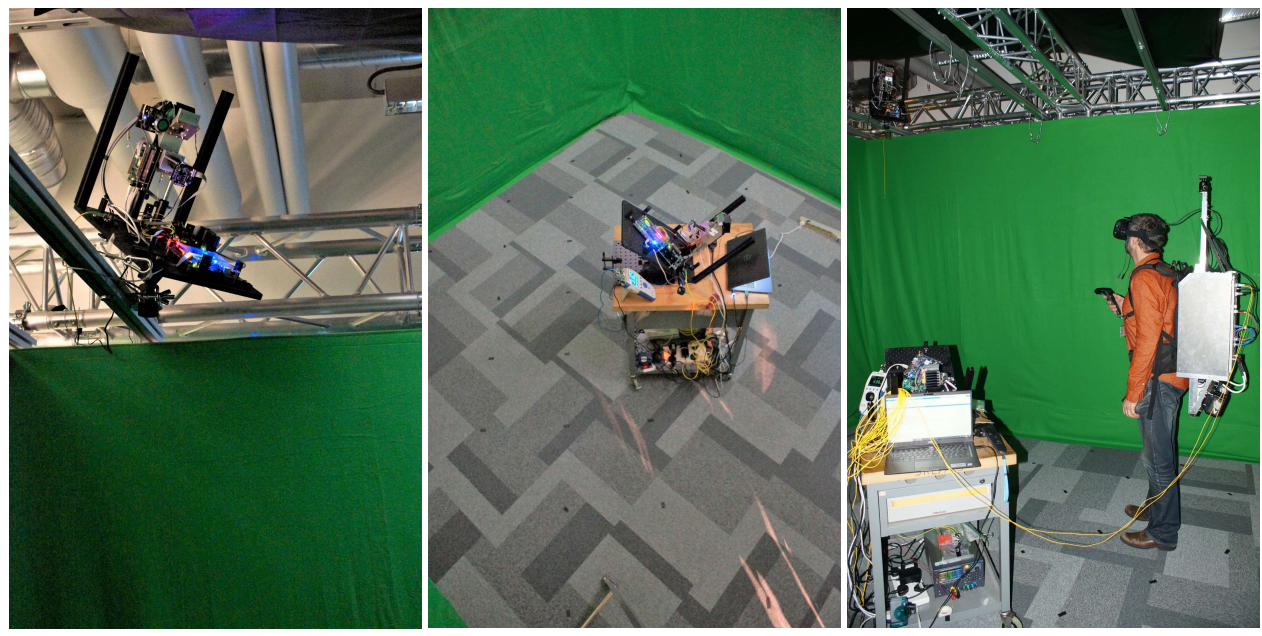

Fig. 17. FWF terminal setup in $4 \times 4 \times 3$ meter indoor testing facility at BCOM laboratory. The AP terminal in one corner at $3.5 \mathrm{~m}$ height (left), the UE terminal on the room floor (centre) and VR-HMD connected to the UE terminal (right).
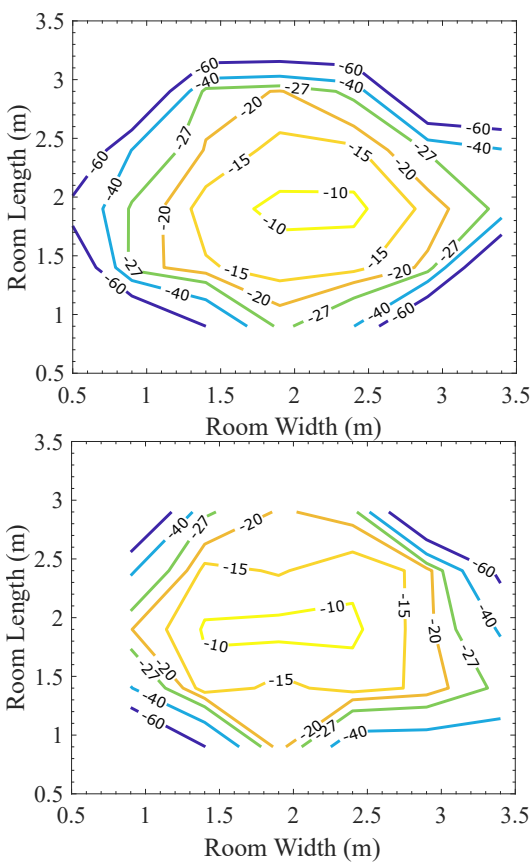

Fig. 18. FWF terminal down-link (top) and up-link (bottom) received power levels $(\mathrm{dBm})$ for $10.3 \mathrm{Gbit} / \mathrm{s}$ free-space transmission when the UE terminal moved across the room floor.

The terminal coverage was evaluated in a $4 \times 4 \times 3$ meter indoor testing facility at the BCOM laboratory in Rennes, France. Fig. 17 shows the setup of terminals within the test facility. One terminal acted as access point (AP), mounted in one corner of the room at a height of $3.5 \mathrm{~m}$. The other terminal acted as user-equipment (UE), which was placed on a trolley (roughly $1.15 \mathrm{~m}$ from the floor) so that it could be moved across the room to study achievable coverage with the 10G SFP+ transceivers.

Both up-link (UL) and down-link (DL) $10.3 \mathrm{Gbit} / \mathrm{s}$ data transmissions were performed and the terminals were aligned using the localisation system for each location of UE. The received power was measured at each terminal when the
UE moved to different locations. The results are shown in Fig. 18, which reveal that the localisation system can provide approximately $4.9 \mathrm{~m}^{2}$ coverage within the $4 \times 4 \times 3$ meter room, beyond which the localisation system is unable to work due to its limited FoV. Additionally, the $10 \mathrm{Gbit} / \mathrm{s}$ UL and DL transmissions, with limited $27 \mathrm{~dB}$ link budget, worked well within $3.14 \mathrm{~m}^{2}$ with BER $<10^{-9}$.
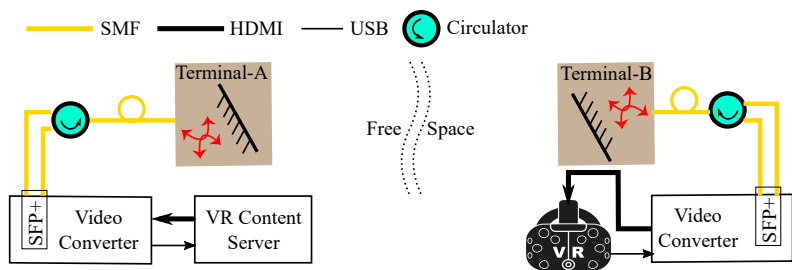

Fig. 19. Experimental setup for data transmission using VR content.

In addition to this, a full-duplex transmission was performed using virtual reality (VR) content. The experimental diagram is shown in Fig.19. The goal was to use a classical VR set up with a wireless link from the VR server to the VR headmounted display (VR-HMD). The DL carried VR content from the VR server to the VR-HMD. A home-made application named "Virtual Arctic Expedition", which provides a visit to sea spaces via virtual diving was used as VR content. On the AP side, a VR server (based on Core i7 PCU + GPU GTX980i) provided this video content as a HDMI signal, with a resolution of $2160 \times 1200$ pixels at $90 \mathrm{~Hz}$ using 8 bits per pixel. The video signal had a raw throughput of $5.6 \mathrm{Gbps}$, which fed a video converter (ArriaX FPGA) via its HDMI input port. The video converter converted the HDMI signal into data flow using 10GETH protocol, which fed an SFP+ transceiver (FTLX1871M3BCL). This SFP+ transceiver was connected to the AP (Terminal-A) via SMF and a circulator (ThorLabs 6015-3-APC) to enable full-duplex communication.

On the UE side, the user terminal (Terminal-B) was connected to another video converter board via SMF and a circulator, where the received Ethernet packets were converted back into the HDMI video signal. This signal was then streamed to 
a VR-HMD (HTC Vive) connected to the video converter via an HDMI cable. The UL was needed to carry the localisation information to the video server from the VR-HMD using the same video converters and optical SFP+ transceivers. The VR-HMD transfers the localisation information to the video converter via a USB link and on the AP side another USB link is used to transfer this information from the video converter to the VR server. Therefore, in the full duplex optical link, the DL carried the video content (5.6Gbps) and the UL carried the localisation information (several Mbps). This localisation information is specifically needed for the VR-HMD, generated by the LightHouse system provided with the HTC Vive and it should not be confused with the localisation and tracking system information for beam-steering in the terminals. The video transmission was tested over different locations within the test facility and no disruption was observed. However, use of additional components (two circulators and four FC/FC connectors) for full-duplex wireless operation introduced approximately $5 \mathrm{~dB}$ additional loss in the UL and DL, which limited the coverage to $1.5 \mathrm{~m}^{2}$ for video transmission.

It is apparent that the data/video transmission coverage can be further increased to $4.9 \mathrm{~m}^{2}$ by increasing the transmit power to the Class-I eye-safety limit and by using optical amplification at the receiver of each terminal. In addition to this, there are several ways to further optimise or improve the performance of the tracking and beam-steering system to minimise the pointing loss. The pointing error is measured by comparing the pointing angles obtained from the localisation $\&$ tracking system to those obtained from optimal alignment. The implemented pointing correction uses the pointing error information obtained from one-dimensional rotation/steering in the horizontal and vertical planes. This one dimensional aberration correction in horizontal and vertical plane (as shown in Fig. 7), minimises the pointing loss very effectively in the respective planes, to close to the values obtained by manually optimised pointing. However, the link losses observed in a two-dimensional steering increase significantly in the available FoV as the link angle increases, as seen in Fig. 18. Similar conclusions can be made from the results in Fig. 13, which shows that manual optimisation provides better BER. This indicates that the horizontal and vertical corrections are not separable, so a full two-dimensional correction mapping is required to obtain similar losses to those achieved using manual optimisation. In addition to this, the OptoTune mirror used in the FWF terminals can be steered with a pointing accuracy of $0.001^{\circ}$. This was verified experimentally where a smaller pointing loss was observed by manually aligning the terminals with such accuracy. However, the measured accuracy of the tracking system is $0.02^{\circ}$. Therefore, there is a room for further improvement in the tracking system performance which can further minimise the pointing loss. A position sensitive detector (rather than a camera) is currently being evaluated as a fine-tracking sensor (which also offers lower latency), and a higher pixel-count camera may also offer a route to higher accuracy.

\section{Conclusions}

The design, implementation and performance evaluation of FWF terminals for OWC with realistic coverage has been presented. Fast dual-axis steering mirrors and small form factor low-cost, low pixel-count cameras have been integrated in a $15 \times 14 \times 13 \mathrm{~cm}$ housing to achieve a $50^{\circ}$ full angle FoV with $0.02^{\circ}$ localisation accuracy. The terminals have a capability to operate over a wide optical spectrum with a low link-loss and have a tracking latency of $200 \mathrm{~ms}$. Through laboratory based experiments, a $1 \mathrm{Tbit} / \mathrm{s}$ transmission capability has been demonstrated. Considering a room size environment, $4.9 \mathrm{~m}^{2}$ localisation coverage has been achieved. Further, using off-the-shelf SFP+ modules, a $10 \mathrm{Gbit} / \mathrm{s}$ link between access and user terminals was demonstrated over a $3.14 \mathrm{~m}^{2}$ coverage. A full-duplex wireless link between a VR server and a VR-HMD using the FWF terminals was also demonstrated, where VR content was streamed to VR-HMD and HMD related localisation information was streamed to VR server in real-time. Future work is focused on increasing the tracking speed to enable terminal mobility.

\section{ACKNOWLEDGEMENT}

This work was supported by the European Union's Horizon 2020 research and innovation program under grant agreement No. 761329 (project WORTECS) and, by the UK EPSRC project COALESCE (EP/P003990/1).

\section{REFERENCES}

[1] E. Agrell, M. Karlsson, A. Chraplyvy, D. J. Richardson, P. M. Krummrich, P. Winzer, K. Roberts, J. K. Fischer et al., "Roadmap of optical communications," Journal of Optics, vol. 18, no. 6, p. 063002, 2016.

[2] F. J. Effenberger, "Industrial trends and roadmap of access," Journal of Lightwave Technology, vol. 35, no. 5, pp. 1142-1146, 2016.

[3] T. Nagatsuma, G. Ducournau, and C. C. Renaud, "Advances in terahertz communications accelerated by photonics," Nature Photonics, vol. 10, no. 6 , p. 371,2016

[4] W. Hong, K.-H. Baek, and S. Ko, "Millimeter-wave 5g antennas for smartphones: Overview and experimental demonstration," IEEE Transactions on Antennas and Propagation, vol. 65, no. 12, pp. 6250-6261, 2017.

[5] H. Chun, A. Gomez, C. Quintana, W. Zhang, G. Faulkner, and D. O'Brien, "A wide-area coverage $35 \mathrm{gb} / \mathrm{s}$ visible light communications link for indoor wireless applications," Scientific reports, vol. 9, no. 1, p. 4952, 2019.

[6] A. Gomez, K. Shi, C. Quintana, R. Maher, G. Faulkner, P. Bayvel, B. C. Thomsen, and D. O'Brien, "Design and demonstration of a 400 $\mathrm{gb} / \mathrm{s}$ indoor optical wireless communications link," Journal of Lightwave Technology, vol. 34, no. 22, pp. 5332-5339, 2016.

[7] A. Gomez, K. Shi, C. Quintana, M. Sato, G. Faulkner, B. C. Thomsen, and D. O'Brien, "Beyond 100-gb/s indoor wide field-of-view optical wireless communications," IEEE Photonics Technology Letters, vol. 27, no. 4, pp. 367-370, 2014.

[8] C. Oh, E. Tangdiongga, and A. Koonen, "Steerable pencil beams for multi-gbps indoor optical wireless communication," Optics letters, vol. 39, no. 18, pp. 5427-5430, 2014.

[9] T. Koonen, F. Gomez-Agis, F. Huijskens, K. A. Mekonnen, Z. Cao, and E. Tangdiongga, "High-capacity optical wireless communication using two-dimensional ir beam steering," Journal of Lightwave Technology, vol. 36, no. 19, pp. 4486-4493, 2018.

[10] L. Gonzalez-Guerrero, H. Shams, I. Fatadin, M. J. Fice, M. Naftaly, A. J. Seeds, and C. C. Renaud, "Comparison of optical single sideband techniques for thz-over-fiber systems," IEEE Transactions on Terahertz Science and Technology, vol. 9, no. 1, pp. 98-105, 2018.

[11] J. Wang, J.-Y. Yang, I. M. Fazal, N. Ahmed, Y. Yan, H. Huang, Y. Ren, Y. Yue, S. Dolinar, M. Tur et al., "Terabit free-space data transmission employing orbital angular momentum multiplexing," Nature photonics, vol. 6 , no. 7, p. 488, 2012. 
[12] H. Huang, G. Xie, Y. Yan, N. Ahmed, Y. Ren, Y. Yue, D. Rogawski, M. J. Willner, B. I. Erkmen, K. M. Birnbaum et al., "100 tbit/s free-space data link enabled by three-dimensional multiplexing of orbital angular momentum, polarization, and wavelength," Optics letters, vol. 39, no. 2, pp. 197-200, 2014.

[13] E. Ciaramella, Y. Arimoto, G. Contestabile, M. Presi, A. D’Errico, V. Guarino, and M. Matsumoto, "1.28 terabit/s (32x40 gbit/s) wdm transmission system for free space optical communications," IEEE Journal on selected areas in communications, vol. 27, no. 9, pp. 16391645, 2009.

[14] P. Brandl, S. Schidl, A. Polzer, W. Gaberl, and H. Zimmermann, "Optical wireless communication with adaptive focus and mems-based beam steering," IEEE Photonics Technology Letters, vol. 25, no. 15, pp. 1428$1431,2013$.

[15] P. Brandl, A. Weiss, and H. Zimmermann, "Automated alignment system for optical wireless communication systems using image recognition," Optics letters, vol. 39, no. 13, pp. 4045-4048, 2014.

[16] S.-R. Cho, K. Lee, M. Kye, and C.-H. Lee, "Cost-effective autoalignment method for indoor optical wireless communication," in Asia Communications and Photonics Conference. Optical Society of America, 2017, pp. M3F-6.

[17] B. Glushko, A. Shar, M. Medina, D. Kin, and S. Krylov, "Mems-based tracking for an indoor optical wireless communication bidirectional link," IEEE Photonics Technology Letters, vol. 28, no. 5, pp. 550-553,
2016.

[18] M. Kavehrad, "Mems-based reconfigurable optical wireless networking in data centers," in 2017 IEEE Photonics Conference (IPC). IEEE, 2017 , pp. $127-128$

[19] K. Wang, A. Nirmalathas, C. Lim, and E. Skafidas, "4 x $12.5 \mathrm{gb} / \mathrm{s}$ wdm optical wireless communication system for indoor applications," Journal of Lightwave Technology, vol. 29, no. 13, pp. 1988-1996, 2011.

[20] A. Gomez, K. Shi, C. Quintana, G. Faulkner, B. C. Thomsen, and D. O'Brien, "A $50 \mathrm{gb} / \mathrm{s}$ transparent indoor optical wireless communications link with an integrated localization and tracking system," Journal of Lightwave Technology, vol. 34, no. 10, pp. 2510-2517, 2016.

[21] F. Feng, P. Sangwongngam, G. Faulkner, and D. O'Brien, "Wide fieldof-view optical broadcasting for bi-directional indoor optical wireless communications employing pam-4 modulation," Optics Letters, vol. 44, no. 24, pp. 6009-6012, 2019.

[22] N. Pham, K. A. Mekonnen, E. Tangdiongga, A. Mefleh, and A. Koonen, "Multi-user localization and upstream signaling for indoor owc system using a camera technology," in Optical Fiber Communication Conference. Optical Society of America, 2020, pp. M1J-7.

[23] Y. Hong, F. Feng, K. R. H. Bottrill, N. Taengnoi, R. Singh, G. Faulkner, D. O'Brien, and P. P., "Beyond terabit/s wdm optical wireless transmission using wavelength-transparent beam tracking and steering," in to appear in OSA Conference on Optical Fiber Communication (OFC). OSA, 2020. 